\title{
Experimental and numerical results on three-dimensional instabilities in a rotating disk-tall cylinder flow
}

\author{
Sørensen, Jens Nørkær; Gelfgat, A. Yu; Naumov, I. V.; Mikkelsen, Robert Flemming
}

Published in:

Physics of Fluids

Link to article, DOI:

$10.1063 / 1.3133262$

Publication date:

2009

Document Version

Publisher's PDF, also known as Version of record

Link back to DTU Orbit

Citation (APA):

Sørensen, J. N., Gelfgat, A. Y., Naumov, I. V., \& Mikkelsen, R. F. (2009). Experimental and numerical results on three-dimensional instabilities in a rotating disk-tall cylinder flow. Physics of Fluids, 21(5), 054102.

https://doi.org/10.1063/1.3133262

\section{General rights}

Copyright and moral rights for the publications made accessible in the public portal are retained by the authors and/or other copyright owners and it is a condition of accessing publications that users recognise and abide by the legal requirements associated with these rights.

- Users may download and print one copy of any publication from the public portal for the purpose of private study or research.

- You may not further distribute the material or use it for any profit-making activity or commercial gain

- You may freely distribute the URL identifying the publication in the public portal 


\title{
Experimental and numerical results on three-dimensional instabilities in a rotating disk-tall cylinder flow
}

\author{
J. N. Sørensen, ${ }^{1}$ A. Yu. Gelfgat, ${ }^{2, a)}$ I. V. Naumov, ${ }^{3}$ and R. F. Mikkelsen ${ }^{1}$ \\ ${ }^{1}$ Department of Mechanical Engineering, Center for Fluid Dynamics, Technical University of Denmark, \\ DK-2800 Lyngby, Denmark \\ ${ }^{2}$ School of Mechanical Engineering, Faculty of Engineering, Tel Aviv University, Tel-Aviv 69978, Israel \\ ${ }^{3}$ Institute of Thermophysics, SB RAS, Novosibirsk 630090, Russia
}

(Received 18 January 2009; accepted 20 April 2009; published online 13 May 2009)

\begin{abstract}
The three-dimensional axisymmetry-breaking instability of axisymmetric flow between a rotating lid and a stationary cylinder is analyzed both numerically and experimentally for the case of tall cylinders with the height/radius aspect ratio between 3.3 and 5.5. A complete stability diagram for the primary three-dimensional instability is obtained experimentally and computed numerically. The instability sets in due to different three-dimensional disturbance modes that are characterized by different azimuthal wavenumbers. The critical Reynolds numbers and associated frequencies are identified for each mode. The onset of three-dimensional flow behavior is measured by combining the high spatial resolution of particle image velocimetry and the temporal accuracy of laser Doppler anemometry. The results are compared to the numerical stability analysis. The measured onset of three dimensionality is in a good agreement with the numerical results. Disagreements observed in supercritical regimes can be explained by secondary bifurcations that are not accounted for by linear stability analysis of the primary base flow. (C) 2009 American Institute of Physics.
\end{abstract}

[DOI: 10.1063/1.3133262]

\section{INTRODUCTION}

Many flows generated by rotation or natural convection in axisymmetric enclosures with axisymmetric boundary conditions break into nonaxisymmetric patterns above a certain threshold of the governing parameters. Such axisymmetry-breaking instabilities are of interest in stability analysis and are of major importance in applications.

Swirling flows in a closed cylindrical container with a rotating lid has been the subject of a large number of experimental and computational studies for more than 3 decades. A wide interest to this configuration was triggered by experiments of Escudier ${ }^{1}$ that were followed by dozens of experimental and numerical studies. Apart from single lid rotation, different variations of the basic configuration, including, e.g., simultaneous rotation of the other end wall or the introduction of different secondary control mechanisms, have been extensively studied in the past years. Unfortunately, all these studies were never properly reviewed in a single review paper. The reader can be addressed to several well known, ${ }^{2-9}$ as well as recently published ${ }^{10-14}$ studies and references therein. The emphasis of the present work, however, is on the "classical" problem of a cylinder with one rotating lid, which probably is the simplest configuration for studying transition and onset of unsteady modes in rotating flow.

The rotating lid drives the fluid around the cylinder axis. The nonuniform centrifugal force pushes the fluid adjacent to the lid away from the center, which results in a meridional circulation. The fluid then descends along the outer wall and turns inward near the fixed bottom. Close to the center axis it

\footnotetext{
${ }^{a)}$ Author to whom correspondence should be addressed. Telephone: 972-3640-7207. Fax: 972-3-640-7334. Electronic mail: gelfgat@eng.tau.ac.il.
}

ascends vertically, forms a concenterd vortex structure due to the conservation of angular momentum, and returns to the rotating lid. At a slow lid rotation the flow is axisymmetric and steady. Depending on the rotation rate and aspect ratio the flow may experience vortex breakdown in the form of recirculating bubbles appearing at the axis of the cylinder. ${ }^{1}$ With an increase in the rotation rate, depending on the aspect ratio, the flow is subject to a complicated transition process in which it becomes unsteady and three dimensional. This process is followed by the development of various threedimensional structures that differ both in space and in time. Changes in the flow structures depend on two parameters: the aspect ratio $h$ (ratio of the container height to the disk radius $H / R$ ) and the Reynolds number $\operatorname{Re}=\Omega R^{2} / \nu$, where $\Omega$ is the angular velocity of the disk and $\nu$ is the kinematic viscosity of the working fluid.

In the paper by Gelfgat et al. ${ }^{6}$ a detailed analysis of the onset of nonaxisymmetric instabilities as a function of Reynolds number was performed for aspect ratios in the range $1 \leq h \leq 3.5$. The analysis was carried out using a spectral Galerkin method combined with an eigenvalue analysis to detect the azimuthal wave numbers, $k$, associated with marginal and critical modes. The marginal and critical Reynolds numbers and associated critical frequencies of the perturbations were computed and several individual instability modes were identified.

To supplement the visualizations of Escudier ${ }^{1}$ and the analysis of Gelfgat et al., ${ }^{6}$ a series of experiments aiming to detect critical modes in the rotating cavity by quantitative and nonintrusive experimental techniques was performed in the paper of Sørensen et al. ${ }^{9}$ A combination of the high spatial resolution of particle image velocimetry (PIV) with the 
temporal accuracy of laser Doppler anemometry (LDA) allowed us to extract modes of the dominant azimuthal waves and to map the transition from steady axisymmetric to unsteady three-dimensional flow. We observed that depending on the aspect ratio, with a gradual increase in the Reynolds number, the flow becomes unsteady and nonaxisymmetric. At a Reynolds number beyond 4500 the oscillations become nonperiodic, which makes it impossible to extract distinct modes and frequencies, as well as to distinguish small-scale fluctuations from noise.

The development of instability was studied experimentally by Naumov et al. ${ }^{10}$ Two scenarios of the instability development in which the amplitude of velocity pulsations grows or decays with increasing swirl of the flow have been observed. It was established that the instability evolution depends on the appearance of secondary perturbations and on their frequency. Another experimental study considering the increasing aspect ratio was performed by Okulov et al. ${ }^{11}$ These experiments determined the boundary of transition to unsteadiness arising as a result of the equilibrium rotation of self-organized vortex multiplets (triplet, double triplet, double doublet, and quadruplet) depending on $h$ considered in a wider interval than that studied previously.

To the best of our knowledge, no detailed analysis of nonaxisymmetric instability for aspect ratio $h \geq 3.5$ has been performed. The main purpose of this study is experimental and numerical investigation of the onset of nonaxisymmetric instabilities in a closed cylinder with rotating lid for aspect ratios $3.3 \leq h \leq 5.5$. The use of PIV allows for accurate simultaneous measurements of the whole velocity field in a two-dimensional plane normal to the cylinder axis. At the same time LDA allows one to measure onset of an unsteady flow and to determine its frequency. Thus, combination of the two techniques yields both the frequency and the azimuthal periodicity of appearing unsteady flow. In the present paper the marginal Reynolds number, $\operatorname{Re}_{m}(h, k)$, is defined as the lowest Reynolds number at which a particular mode with azimuthal wave number $k$ starts to grow. A marginal frequency denoted as $\omega_{m}(h, k)$ is associated with each mode having a particular wavenumber $k$. The critical Reynolds number was defined as $\operatorname{Re}_{\text {cr }}(h)=\min _{k} \operatorname{Re}_{m}(h, k)$ so that the most unstable mode can be recognized as one corresponding to the lowest $\operatorname{Re}_{m}(h, k)$. We begin by determining the marginal and critical Reynolds numbers at which the basic steady and axisymmetric state loses its stability. Then we perform thorough measurements in which different azimuthal modes and frequencies are detected as function of wave number, Reynolds numbers, and aspect ratio.

The experimental setup, the measurement techniques and data processing methods used in this work were described in Ref. 9 and the numerical simulation method was presented in Ref. 15. Below we report a detailed mapping of critical Reynolds numbers, characteristic frequencies, and azimuthal modes, depicted as functions of aspect ratio. A good agreement between the experimental and numerical results yields the validation of numerical technique, as it was recently pointed out by Roache. ${ }^{16}$

\section{ONSET OF AZIMUTHAL MODES AND CHARACTERISTIC FREQUENCIES}

The LDA measurements were systematically carried out in the range $h \in[3.3,5.5]$, starting from $h=3.3$ with an increment of $\Delta h=0.1$, and in the range $\operatorname{Re} \in[2000,5000]$, starting from $\operatorname{Re}=2000$ and with an increment $\Delta R e=100$. The experimentally observed transitions are summarized in Table I, which displays the occurrence of marginal and critical Reynolds numbers and associated frequencies, as function of the aspect ratio $h \in[3.3,5.5]$ and the wave number $k \in[0,5]$. The frequency $\omega_{k}$ is scaled by the rotational frequency of the lid $\Omega$. The linear stability analysis defines a signed value of $\omega_{k}$, for which positive or negative values correspond to the co- or counterpropagation of azimuthal traveling wave with respect to rotation of the lid. The numerical study predicts that the modes $k=0$ and 1 correspond to the copropagation, while higher modes cause the counterpropagation of azimuthal wave. In the LDA measurements the sign of $\omega_{k}$ is indefinite so that only absolute value of $\omega_{k}$ can be used for comparison. To distinguish between marginal and critical Reynolds numbers, the critical values are designated in Table I by bold numbers. Based on the information gained from the LDA-measured time histories, the time ranges for averaging of instantaneous velocity fields are determined for the PIV method. The resulting perturbed velocity fields were derived by averaging over a series of realizations at divisible time periods and then subtracting the mean flow field. Since a mode with the same wavenumber may appear with different frequencies at different Reynolds numbers, the table in some cases displays two modes for the same mode number, e.g., 3 and $3^{\prime}$.

The observed marginal and critical Reynolds numbers, and associated frequencies, are plotted in Figs. 1 and 2 and compared to the numerical results. It follows from Fig. 1 that the azimuthal mode $k=3$ is the dominant one in the range $3.3<h<4$.2. In the range $4.2<h<5.2$ our measurements show that $k=2$ is the most unstable mode and that $k=4$ is the most unstable mode for $5.2<h<5.5$. Thus, for the range of the aspect ratios considered the instability is three dimensional. Note that Serre and Bontoux ${ }^{7}$ found numerically that at $h=4$ the instability sets in as an axisymmetric $(k=0)$ mode at $\operatorname{Re}=3500$. This result agrees with the marginal Reynolds number calculated here for the axisymmetric mode; however, there exist four more unstable modes with $k=2,3,4$ and 5, which are observed here both experimentally and numerically and were overlooked in the calculations in Ref. 7. The reason for that can be either an unsuccessful attempt to find the correct instability threshold by time-dependent calculations, which cannot include all possible perturbations, or insufficient spatial resolution as is discussed in Ref. 15.

Comparison between the experimental and numerical results must be divided into two parts: comparison of the critical values for critical modes and comparison of the marginal Reynolds numbers and associated frequencies corresponding to other azimuthal modes. The experimental and numerical results for critical Reynolds numbers (Fig. 1), as well as critical frequencies (Fig. 2), exhibit an excellent agreement. The critical curve is zoomed in Fig. 3. There we observe that 
TABLE I. Critical and marginal Reynolds numbers and associated nondimensional frequencies for varying azimuthal wavenumber and aspect ratio. The critical numbers are shown in bold.

\begin{tabular}{|c|c|c|c|c|c|c|c|c|c|c|c|}
\hline & & 3.3 & 3.4 & 3.5 & 3.6 & 3.7 & 3.8 & 3.9 & 4.0 & 4.1 & 4.2 \\
\hline \multirow[t]{2}{*}{$k=0$} & $\mathrm{Re}$ & 3100 & 3100 & 3100 & 3200 & 2900 & 2900 & 2900 & 3200 & 3100 & 3000 \\
\hline & $\left|\omega_{k}\right|$ & 0.15 & 0.14 & 0.14 & 0.14 & 0.14 & 0.14 & 0.13 & 0.12 & 0.12 & 0.11 \\
\hline \multirow[t]{2}{*}{$k=1$} & $\mathrm{Re}$ & 4000 & 4000 & 3900 & 4000 & 3900 & 4000 & 4200 & 3900 & 4000 & \\
\hline & $\left|\omega_{k}\right|$ & 0.07 & 0.07 & 0.07 & 0.10 & 0.09 & 0.09 & 0.08 & 0.08 & 0.08 & \\
\hline \multirow[t]{2}{*}{$k=2$} & $\mathrm{Re}$ & 4000 & 4100 & 4200 & 4400 & 2800 & 2600 & 2900 & 2800 & 2800 & 2700 \\
\hline & $\left|\omega_{k}\right|$ & 0.03 & 0.03 & 0.03 & 0.06 & 0.06 & 0.19 & 0.16 & 0.16 & 0.17 & 0.16 \\
\hline \multirow[t]{2}{*}{$k=3$} & $\operatorname{Re}$ & 2100 & 2100 & 2100 & 2100 & 2100 & 2200 & 2200 & 2300 & 2300 & 2400 \\
\hline & $\left|\omega_{k}\right|$ & 0.31 & 0.31 & 0.29 & 0.28 & 0.27 & 0.27 & 0.27 & 0.27 & 0.27 & 0.26 \\
\hline \multirow{2}{*}{$k=3^{\prime}$} & $\operatorname{Re}$ & 2700 & 2200 & & & & & & & & \\
\hline & $\left|\omega_{k}\right|$ & 0.29 & 0.29 & & & & & & & & \\
\hline \multirow{2}{*}{$k=4$} & $\operatorname{Re}$ & 3500 & 3500 & 3400 & 3400 & 3200 & 3400 & 2800 & 2600 & 2800 & 3000 \\
\hline & $\left|\omega_{k}\right|$ & 0.43 & 0.42 & 0.41 & 0.41 & 0.41 & 0.39 & 0.39 & 0.38 & 0.39 & 0.37 \\
\hline \multirow[t]{2}{*}{$k=4^{\prime}$} & $\mathrm{Re}$ & 2900 & 2900 & 3000 & 3000 & 2800 & 2700 & & & & \\
\hline & $\left|\omega_{k}\right|$ & 0.39 & 0.38 & 0.37 & 0.35 & 0.35 & 0.34 & & & & \\
\hline \multirow[t]{3}{*}{$k=5$} & $\operatorname{Re}$ & 3400 & 3100 & 3100 & 3300 & 3200 & 3200 & 3100 & 2800 & 3100 & 3200 \\
\hline & $\left|\omega_{k}\right|$ & 0.54 & 0.53 & 0.52 & 0.52 & 0.54 & 0.52 & 0.52 & 0.47 & 0.49 & 0.49 \\
\hline & & 4.4 & 4.6 & 4.8 & 4.9 & 5.0 & 5.1 & 5.2 & 5.3 & 5.4 & 5.5 \\
\hline \multirow[t]{2}{*}{$k=0$} & $\mathrm{Re}$ & 3400 & 3600 & 3900 & 4100 & 3900 & 3900 & 4200 & 4300 & 4300 & 4400 \\
\hline & $\left|\omega_{k}\right|$ & 0.10 & 0.10 & 0.09 & 0.10 & 0.11 & 0.11 & 0.07 & 0.07 & 0.10 & 0.07 \\
\hline \multirow[t]{2}{*}{$k=1$} & $\mathrm{Re}$ & 3900 & 4200 & 4200 & 4500 & 4200 & 4700 & & 4700 & 4500 & 4500 \\
\hline & $\left|\omega_{k}\right|$ & 0.08 & 0.07 & 0.07 & 0.08 & 0.08 & 0.08 & & 0.04 & 0.05 & 0.04 \\
\hline \multirow[t]{2}{*}{$k=2$} & $\operatorname{Re}$ & 2600 & 2700 & 2900 & 2900 & 3000 & 3100 & 3000 & 3200 & 3200 & 3300 \\
\hline & $\left|\omega_{k}\right|$ & 0.15 & 0.14 & 0.14 & 0.13 & 0.13 & 0.13 & 0.11 & 0.13 & 0.13 & 0.13 \\
\hline \multirow[t]{2}{*}{$k=3$} & $\operatorname{Re}$ & 2600 & 2800 & 3000 & 3100 & 3200 & 3200 & 3200 & 3400 & 3500 & 3400 \\
\hline & $\left|\omega_{k}\right|$ & 0.27 & 0.25 & 0.25 & 0.25 & 0.23 & 0.24 & 0.23 & 0.23 & 0.23 & 0.23 \\
\hline \multirow[t]{2}{*}{$k=3^{\prime}$} & $\mathrm{Re}$ & & & & & & & & 4400 & 4400 & \\
\hline & $\left|\omega_{k}\right|$ & & & & & & & & 0.27 & 0.26 & \\
\hline \multirow[t]{2}{*}{$k=4$} & $\operatorname{Re}$ & 2900 & 3000 & 3200 & 3200 & 3300 & 3300 & 3100 & 3100 & 3100 & 2900 \\
\hline & $\left|\omega_{k}\right|$ & 0.38 & 0.37 & 0.36 & 0.35 & 0.35 & 0.37 & 0.37 & 0.38 & 0.37 & 0.37 \\
\hline \multirow[t]{2}{*}{$k=4^{\prime}$} & $\operatorname{Re}$ & & & & & & 4100 & 4200 & 4300 & 4400 & 4000 \\
\hline & $\left|\omega_{k}\right|$ & & & & & & 0.31 & 0.32 & 0.31 & 0.31 & 0.31 \\
\hline \multirow[t]{2}{*}{$k=5$} & $\operatorname{Re}$ & 3400 & 3400 & 3500 & 3300 & 3200 & 3200 & 3200 & 3300 & 3400 & 3500 \\
\hline & $\left|\omega_{k}\right|$ & 0.48 & 0.46 & 0.44 & 0.45 & 0.45 & 0.47 & 0.47 & 0.47 & 0.47 & 0.45 \\
\hline \multirow[t]{2}{*}{$k=5^{\prime}$} & $\operatorname{Re}$ & & & & & & 4400 & 4200 & 4300 & 4500 & 4800 \\
\hline & $\left|\omega_{k}\right|$ & & & & & & 0.42 & 0.42 & 0.41 & 0.41 & 0.39 \\
\hline
\end{tabular}




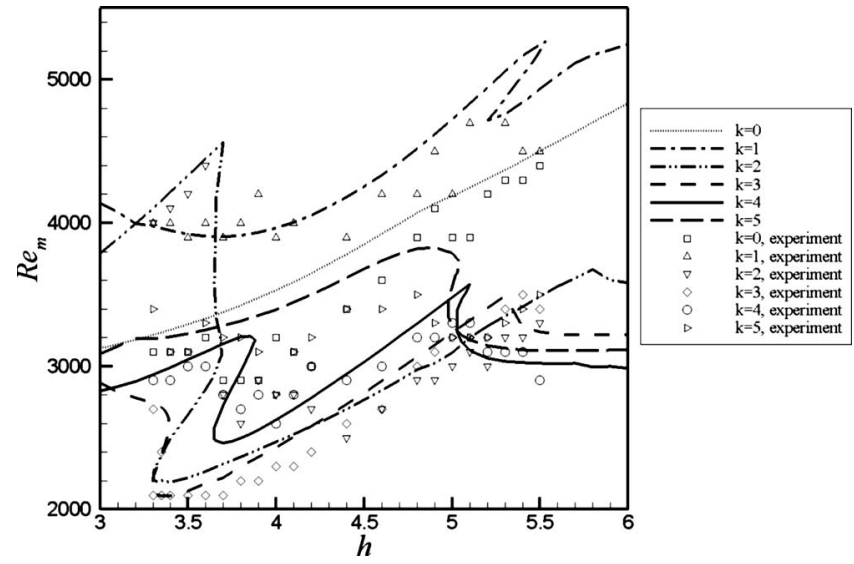

FIG. 1. Marginal Reynolds numbers as function of aspect ratio and wave number.

experimentally measured critical $\operatorname{Re}$ for $3.5<h<5$ are slightly below those predicted numerically. This can be a consequence of a subcritical bifurcation to three dimensionality, which is yet to be explored. The analysis of the bifurcation direction was beyond the scope of present study.

The agreement between experimental and numerical marginal Reynolds numbers for other, noncritical modes is also good but apparently not so striking (Fig. 1). This is not surprising since the onset of these modes was measured for already unstable flow so that the base flow already was three dimensional. In fact the experimental measurements correspond rather to the secondary, tertiary, and higher bifurcations, while the numerical results correspond to the primary one. In view of this the agreement is not only good but also surprising. Not less surprising is the agreement between the measured and calculated frequencies (Fig. 2). In our opinion this agreement indicates that in spite of already developed break of axial symmetry the asymmetric part of the flow remains much weaker than its axisymmetric part. Thus it is likely that the mechanisms responsible for instability of the axisymmetric flow prevail.

In the numerical simulations only the lowest marginal Reynolds numbers were determined for each of the analyzed azimuthal modes. Thus, the computations did not reveal fur-

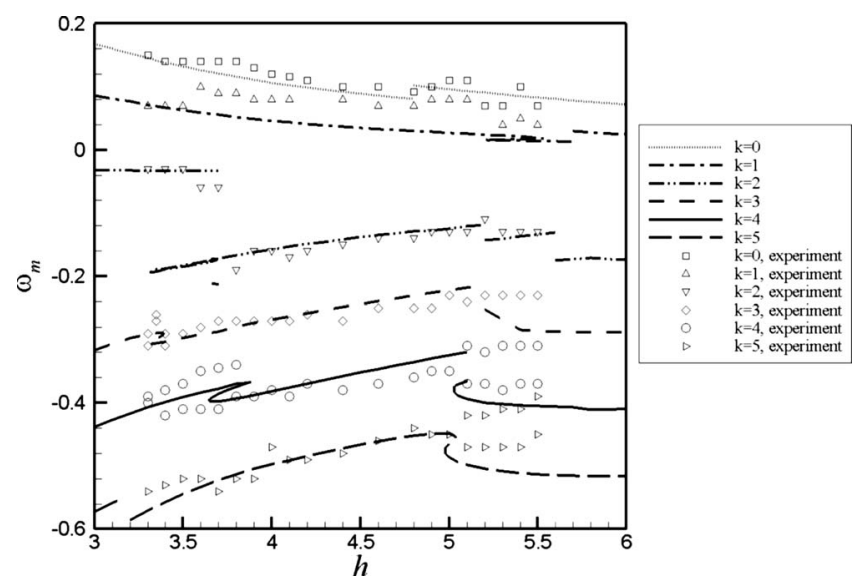

FIG. 2. Marginal frequencies as function of aspect ratio and wave number.

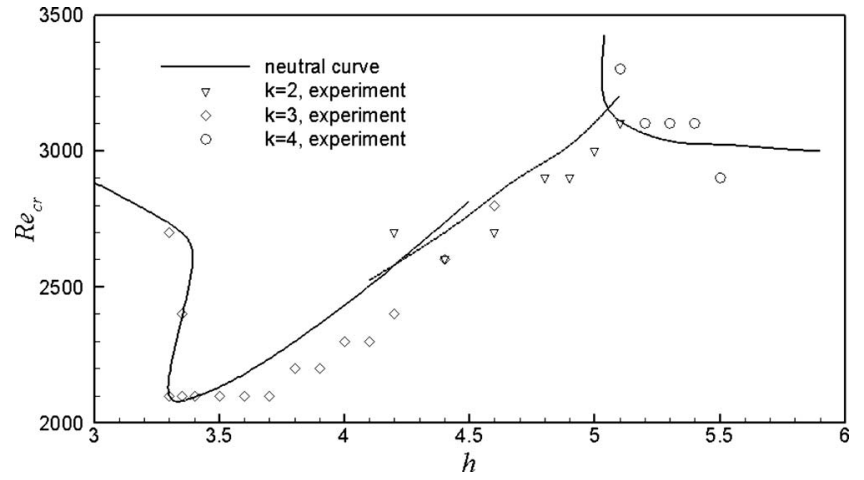

FIG. 3. Critical Reynolds numbers versus the aspect ratio.

ther bifurcations within the same azimuthal symmetry that take place with the increase of the Reynolds number. The experimental technique, however, allows us to follow the modes and their associated frequency as the Reynolds number is increased. Therefore, in some cases, especially at high Reynolds numbers for $5.1<h<5.5$ and for modes $k=3,4$, and 5, the measurements revealed frequencies that were not reflected by the linear stability computations. At large Reynolds numbers all the investigated modes can coexist, indicating that the flow is approaching a chaotic state. Generally, the spectral LDA plots show that the axisymmetric mode $k$ $=0$ is most energetic near the cylinder axis, and that the other modes, $k=2,3$, or 4 , are most dominant near the sidewalls of the cylinder.

An interesting phenomenon appears for $h=3.3$. Here the most unstable mode, $k=3$, first appears at $\mathrm{Re}=2100$ but stabilizes at $\mathrm{Re}=2300$. Hence, in the range from 2300 to 2700 , the flow again becomes steady and axisymmetric. This phenomenon is observed independently by computations and experiment.

\section{CONCLUSION}

The primary axisymmetry-breaking oscillatory instability of the flow in a closed rotating lid cylinder for aspect ratios $h \in[3.3,5.5]$ was investigated. The marginal and critical Reynolds numbers together with associated frequencies of the perturbed velocity field were determined. The critical and marginal Reynolds numbers, azimuthal periodicities, and characteristic frequencies of the velocity were extracted and compared to the corresponding numerical results. It is found that the nonaxisymmetric instability dominates in whole range $3.3<h<5.5$ considered. The azimuthal mode $k=3$ is the dominant one in the range $3.3<h<4$.2. In the range $4.2<h<5.2$ the computations and measurements show that $k=2$ is the most unstable mode, while $k=4$ is the most unstable mode for $5.2<h<5.5$.

Perfect agreement between calculated and measured characteristics cross validates both experimental and numerical approaches and demonstrates the efficiency of using the new diagnostics technique to analyze oscillatory flows. 


\section{ACKNOWLEDGMENTS}

This research was supported partly by the RFBR (Grant Nos. 07-01-00223 and 07-08-00296, to I. Naumov) and partly by the German-Israeli Foundation under Grant No. 954-34.10/2007 (to A. Gelfgat).

${ }^{1}$ M. P. Escudier, "Observations of the flow produced in a cylindrical container by a rotating endwall," Exp. Fluids 2, 189 (1984).

${ }^{2}$ J. M. Lopez, “Axisymmetric vortex breakdown. Part 1: Confined swirling flow," J. Fluid Mech. 221, 533 (1990).

${ }^{3}$ J. M. Lopez and A. D. Perry, "Axisymmetric vortex breakdown. Part 3: Onset of periodic flow and chaotic advection," J. Fluid Mech. 234, 449 (1992).

${ }^{4}$ A. Yu. Gelfgat, P. Z. Bar-Yoseph, and A. Solan, "Stability of confined swirling flow with and without vortex breakdown," J. Fluid Mech. 311, 1 (1996).

${ }^{5}$ A. Yu. Gelfgat, P. Z. Bar-Yoseph, and A. Solan, "Steady states and oscillatory instability of swirling flow in a cylinder with rotating top and bottom," Phys. Fluids 8, 2614 (1996).

${ }^{6}$ A. Yu. Gelfgat, P. Z. Bar-Yoseph, and A. Solan, "Three-dimensional instability of axisymmetric flow in rotating lid-cylinder enclosure," J. Fluid Mech. 438, 363 (2001).

${ }^{7}$ E. Serre and P. Bontoux, "Vortex breakdown in a three-dimensional swirling flow," J. Fluid Mech. 459, 347 (2002).
${ }^{8}$ C. Nore, M. Tartar, O. Daube, and L. S. Tuckerman, "Survey of instability thresholds of flow between exactly counter-rotating disks," J. Fluid Mech. 511, 45 (2004).

${ }^{9}$ J. N. Sørensen, I. Naumov, and R. Mikkelsen, "Experimental investigation in three-dimensional flow instabilities in a rotating lid-driven cavity," Exp. Fluids 41, 425 (2006).

${ }^{10}$ I. V. Naumov, V. L. Okulov, and J. N. Sørensen, "Two scenarios of instability development in flow with strong swirling," Tech. Phys. Lett. 33, 775 (2007).

${ }^{11}$ V. L. Okulov, I. V. Naumov, and J. N. Sørensen, "Self-organized vortex multiplets in swirling flow," Tech. Phys. Lett. 34, 675 (2008).

${ }^{12}$ J. M. Lopez, Y. D. Cui, F. Marques, and T. T. Lim, "Quenching of vortex breakdown oscillations via harmonic modulation," J. Fluid Mech. 599, 441 (2008).

${ }^{13}$ Y. D. Cui, J. M. Lopez, T. T. Lim, and F. Marques, "Harmonically forced enclosed swirling flow," Phys. Fluids 21, 034106 (2009).

${ }^{14}$ B. T. Tan, K. Y. S. Liow, L. Mununga, M. C. Thompson, and K. Hourigan, "Simulation of the control of vortex breakdown in a closed cylinder using a small rotating disk," Phys. Fluids 21, 024104 (2009).

${ }^{15}$ A. Yu. Gelfgat, "Three-dimensional instability of axisymmetric flows: Solution of benchmark problems by a low-order finite volume method," Int. J. Numer. Methods Fluids 54, 269 (2007).

${ }^{16}$ P. J. Roache, "Perspective: Validation-What does it mean?" ASME J. Fluids Eng. 131, 034503 (2009). 